\title{
Nomogram to predict survival of patients with advanced and metastatic pancreatic Cancer
}

\author{
G. C. Deng ${ }^{1,2 \dagger}$, Y. Lv ${ }^{1 \dagger}$, H. Yan ${ }^{1 \dagger}$, D. C. Sun ${ }^{1}$, T. T. Qu ${ }^{1,2}$, Y. T. Pan ${ }^{1}$, Q. L. Han ${ }^{1 *}$ and G. H. Dai ${ }^{1,2^{*}}$
}

\begin{abstract}
Background: Nomograms are rarely employed to estimate the survival of patients with advanced and metastatic pancreatic cancer (PC). Herein, we developed a comprehensive approach to using a nomogram to predict survival probability in patients with advanced and metastatic PC.

Methods: A total of 323 patients with advanced and metastatic PC were identified from the Chinese People's Liberation Army (PLA) General Hospital. A baseline nomogram was constructed using baseline variables of 323 patients. Additionally, 233 patients, whose tumors showed initial responses to first-line chemotherapy, were enrolled in the chemotherapy response-based model. 128 patients and 108 patients with advanced and metastatic PC from January 2019 to April 2021 were selected for external validating baseline model and chemotherapy response-based model. The 1-year and 2-year survival probability was evaluated using multivariate COX regression models. The discrimination and calibration capacity of the nomograms were assessed using C-statistic and calibration plots. The predictive accuracy and net benefit of the nomograms were evaluated using ROC curve and DCA, respectively.

Results: In the baseline model, six variables (gender, KPS, baseline TB, baseline N, baseline WBC and baseline CA19-9) were used in the final model. In the chemotherapy response-based model, nine variables (KPS, gender, ascites, baseline $\mathrm{N}$, baseline CA 19-9, baseline CEA, change in CA 19-9 level at week, change in CEA level at week and initial response to chemotherapy) were included in the final model. The C-statistics of the baseline nomogram and the chemotherapy response-based nomogram were $0.67(95 \% \mathrm{Cl}, 0.62-0.71)$ and 0.74 (95\% Cl, 0.69-0.77), respectively.
\end{abstract}

Conclusion: These nomograms were constructed to predict the survival probability of patients of advanced and metastatic PC. The baseline model and chemotherapy response-based model performed well in survival prediction.

Keywords: Nomogram, Pancreatic cancer, Prognosis, Laboratory parameters, Clinicopathological factors

\section{Introduction}

Pancreatic cancer (PC), a malignancy of the digestive system, shows high mortality. Indeed, PC is the seventh leading cause of cancer-related deaths worldwide [1]. In China, PC is the sixth leading cause of cancer-related mortality [2]. Currently, the 5-year survival rate of

\footnotetext{
*Correspondence: hanquanli@aliyun.com; daigh301@vip.sina.com

${ }^{\dagger}$ G.C Deng, Y LV and H Yan contributed equally as co-first authors.

'School of Medicine, Nankai University, Tianjin, China

Full list of author information is available at the end of the article
}

patients with $\mathrm{PC}$ is below 7\% [3]. Comprehensive chemotherapy-based treatment regimens are the primary choice for patients with advanced or metastatic PC. The preferred chemotherapy regimens recommended by the guidelines of the National Comprehensive Cancer Network (NCCN) include FOLFIRINOX (5-FU), leucovorin, irinotecan, and oxaliplatin, or gemcitabine plus albuminbound paclitaxel for patients with good performance status; gemcitabine monotherapy or 5-FU/capecitabine is used for patients with poor performance status [4]. The median overall survival time of patients receiving these

(c) The Author(s). 2021 Open Access This article is licensed under a Creative Commons Attribution 4.0 International License, which permits use, sharing, adaptation, distribution and reproduction in any medium or format, as long as you give appropriate credit to the original author(s) and the source, provide a link to the Creative Commons licence, and indicate if changes were made. The images or other third party material in this article are included in the article's Creative Commons licence, unless indicated otherwise in a credit line to the material. If material is not included in the article's Creative Commons licence and your intended use is not permitted by statutory regulation or exceeds the permitted use, you will need to obtain permission directly from the copyright holder. To view a copy of this licence, visit http://creativecommons.org/licenses/by/4.0/ The Creative Commons Public Domain Dedication waiver (http://creativecommons.org/publicdomain/zero/1.0/) applies to the data made available in this article, unless otherwise stated in a credit line to the data. 
chemotherapeutic regimens is currently less than one year [5].

The effects of chemotherapy vary in patients with advanced or metastatic PC; therefore, evaluating the prognosis of patients under different conditions is essential for both patients and oncologists. Previous studies have shown that certain risk factors are associated with survival [6-9]. Most predictive models can select some of the individual predictive factors, but cannot calculate survival probability [10-12], which is important to both patients and physicians.

Nomograms, employed to evaluate the survival probability of patients using a specific scoring system $[13,14]$, have been used to predict survival rates in patients with different cancers $[15,16]$. However, few studies have used nomograms to predict the survival rate of patients with advanced or metastatic PC $[17,18]$. Most models are constructed using baseline clinicopathological factors [19]. However, patient responses and sensitivity to chemotherapy are also crucial for constructing predictive models for patients undergoing chemotherapy.

In this study, we aimed to develop a baseline model, constructed using baseline factors, and a chemotherapy response-based model, constructed using factors involved in the initial response to chemotherapy and changes in the expression levels of tumor markers after two cycles of chemotherapy.

\section{Patients and methods}

\section{Patient population}

This study included patients with advanced and metastatic PC who underwent first-line chemotherapy from January 2010 to December 2018 at the Chinese People's Liberation Army (PLA) General Hospital. Follow-up evaluations were performed every 6 months by telephone or by evaluation of medical records.

The inclusion criteria were as follows: (1) diagnosis was confirmed by histopathology or cytological evaluation (2); Karnofsky performance status (KPS) score $\geq$ 70 (3); no previous treatment using first-line chemotherapy (3); evaluation using computed tomography (CT) was conducted before the start of first-line chemotherapy (4); baseline information was collected before first-line chemotherapy (5); availability of CT scans at 6-weeks (before the third cycle of chemotherapy) (6); laboratory factors at 6 weeks, obtained before the third cycle of chemotherapy (7); having explicit terminal status.

From January 2019 to April 2021, an independent cohort of patients with advanced and metastatic PC who underwent first-line chemotherapy was prospectively studied, using the same inclusion and exclusion criteria. This independent group was used for validation cohort of this study.
In the train group, according to these criteria, $323 \mathrm{pa}$ tients were selected into the group used to construct the baseline model. Among these patients, 233 patients, whose tumors had shown initial responses to first-line chemotherapy, were enrolled into the group used to construct the chemotherapy response-based model; these patients had been evaluated using CT scans before the third cycle of chemotherapy. In the validation group, 128 patients and 108 patients were selected for validating baseline model and chemotherapy response-based model. The time point of 6 weeks from the beginning of first-line chemotherapy was selected because chemotherapy regimens were administered using a 3-week cycle, and the evaluation was conducted before the start of third-cycle chemotherapy. Our present study included 134 patients in the train group, treated with nabpaclitaxel plus S-1 regimen in a NPSPAC clinical trial; this was a single-arm, single-center, phase II trial conducted at the Chinese People's Liberation Army (PLA) General Hospital (https://clinicaltrials.gov/ number, NCT02124317).

\section{Clinicopathological variables}

The following demographic and clinicopathological variables were collected: gender, age, body mass index (BMI), KPS, smoking status, use of alcohol, diabetes, jaundice, ascites, metastatic sites, total number of organs with metastases, and location of the primary tumor. The following laboratory values were collected: white blood cell (WBC), platelet (PLT), and neutrophil (N) count; and the levels of albumin (Alb), lactate dehydrogenase (LDH), total bilirubin (TB), serum carcinoembryonic antigen (CEA), and serum carbohydrate antigen 19-9 (CA 19-9). We also examined the levels of serum albumin, LDH, total bilirubin, CEA, and CA 19-9 at the 6week time point. Cancerous lesions were assessed using CT scans before the first cycle, and after two cycles, of chemotherapy. Efficacy of chemotherapy was evaluated using Response Evaluation Criteria In Solid Tumors (RECIST, version 1.0), and patients were classified into the progressive disease (PD) group or non-progressive disease (non-PD) group according to tumor responses. Changes in tumor marker levels at 6 weeks were defined as (value measured at 6 weeks minus baseline value) divided by (baseline value); for example, a change in LDH value at 6 weeks $=([\mathrm{LDH}$ at week 6$]-[\mathrm{LDH}$ at baseline $])$ / ( $\mathrm{LDH}$ at baseline). Based on the obtained values, patients were divided into two categories: the value of $\geq 0$ was defined as the no change and increase group, while the value of $<0$ was defined as the decrease group.

\section{Statistical analysis}

Continuous predictors were expressed using medians with interquartile ranges (IQRs), and categorical predictors 
Table 1 Patient characteristics

\begin{tabular}{|c|c|c|c|c|c|}
\hline \multirow[t]{2}{*}{ Characteristic } & $\begin{array}{l}\text { Baseline } \\
\text { model }\end{array}$ & $\begin{array}{l}\text { Chemotherapy } \\
\text { response-based } \\
\text { model }\end{array}$ & \multirow[t]{2}{*}{ Characteristic } & \multirow{2}{*}{$\begin{array}{l}\begin{array}{l}\text { Baseline } \\
\text { model }\end{array} \\
\text { N Percent (\%) }\end{array}$} & \multirow{2}{*}{$\begin{array}{l}\text { Chemotherapy } \\
\text { response-based } \\
\text { model } \\
\text { N Percent (\%) }\end{array}$} \\
\hline & N Percent (\%) & N Percent (\%) & & & \\
\hline Gender & & & LDH at week 6 & $\mathrm{NI}$ & \\
\hline Female & $125(38.7)$ & $89(38.2)$ & $\leq 170.7$ & & $113(48.5)$ \\
\hline Male & $198(61.3)$ & $144(61.9)$ & $>170.7$ & & $120(51.5)$ \\
\hline Age (year) & & & CEA at week 6 & $\mathrm{NI}$ & \\
\hline$\leq 56$ & $169(52.3)$ & $129(55.4)$ & $\leq 7.97$ & & $117(50.2)$ \\
\hline$>56$ & $154(47.7)$ & $104(44.6)$ & $>7.97$ & & $116(49.8)$ \\
\hline KPS & & & CA $19-9$ at week 6 & NI & \\
\hline $70 \sim 80$ & $95(29.4)$ & $86(36.9)$ & $\leq 790.7$ & & $114(48.9)$ \\
\hline $90 \sim 100$ & $228(70.6)$ & $147(63.1)$ & $>790.7$ & & $119(51.1)$ \\
\hline Number of organ metastases & & & Alb level at week 6 & NI & \\
\hline 0 & $55(17.0)$ & $33(14.2)$ & $\leq 39.0$ & & $117(50.2)$ \\
\hline 1 & $195(60.4)$ & $145(62.2)$ & $>39.0$ & & $116(49.8)$ \\
\hline$\geq 2$ & $73(22.6)$ & $55(23.6)$ & Initial response to chemotherapy & NI & \\
\hline Liver metastases & & & PD & & 55 (23.6) \\
\hline Absent & $74(22.9)$ & $48(20.6)$ & Non-PD & & $178(76.4)$ \\
\hline
\end{tabular}

Baseline WBC $(\times 1000 / \mathrm{mm} 3)$

$$
\leq 6.39
$$$$
>6.39
$$

\section{Baseline N}

$\leq 0.665$

$>0.665$

\section{Baseline LDH}

$$
\leq 159
$$$$
>159
$$

\section{Baseline CEA}

$$
\leq 7.32
$$$$
>7.32
$$

\section{Baseline CA 19-9}

$$
\leq 1453
$$$$
>1453
$$

\section{Change in LDH level at week 6}

Decrease

No change or increase

\section{Change in CEA level at week 6}

Decrease

$162(50.2)$

$161(49.8)$

$162(50.2)$

$161(49.8)$

$155(48.0)$

$168(52.0)$

$161(49.8)$

$162(50.2)$

$115(49.4)$

$118(50.6)$

$162(50.2) \quad 112(48.1)$

161 (49.8)

$121(51.9)$

NI

80 (34.3)

$153(65.7)$

$93(39.9)$

No change or increase

Change in CA 19-9 level at week $\mathrm{NI}$

6

Decrease

$151(64.8)$

No change or increase
Table 1 Patient characteristics (Continued)

were described using counts and proportions. Continuous variables (i.e., age) were categorized into two groups according to their median levels. Correlation of variables was evaluated using a correlation matrix. Overall survival (OS) was defined as the time interval beginning at the date of commencement of first-cycle chemotherapy to the date of death or final follow-up time. We firstly utilized univariate Cox regression to screen for survival related variables. Then, we used backward stepwise selection with Akaike information criterion (AIC) to further select variables. Based on the results of COX multivariate analysis, statistically significant variables were enrolled into the nomograms to predict the probability of survival using the rms package in R. Each nomogram was based on proportionally converting the regression coefficient of each independent risk factor in multivariate COX regression to a number on a 0 - to 100 point scale. The points for each independent variable were added together to derive the total-point score for the predicted probability.

Performance of the predictive model was evaluated using a concordance statistic (C statistic) and calibration. The $\mathrm{C}$-statistic is equal to the area under the receiver operating characteristic (ROC) curve. A C-statistic value of 0.5 indicates no predictive discrimination, while that of 1.0 indicates perfect separation of patients with different outcomes. Calibration with 1000 bootstrap samples to reduce overfitting was estimated by calibration plot. In a perfectly calibrated model, the prediction curve can coincide with the 45-degree diagonal line. The accuracy of the predictive model was evaluated by the ROC curve 
using the timeROC package in $\mathrm{R}$. The net benefit of the model was assessed using decision curve analysis (DCA). We calculated the total scores of patients predicted by the nomograms, used X-tile version 3.6.1 (Yale University, New Haven, CT, USA) to determine the optimal cut-off value for stratifying patients, and performed Kaplan-Meier survival analysis (SPSS 26.0). All statistical analyses were performed in R (version 3.6.3), SPSS26.0, X-tile (version 3.6.1). $p<0.05$ was considered statistically significant.

\section{Results}

\section{Clinical characteristics of the patients}

Among the 323 patients, median overall survival (mOS) was 10.6 months (95\% CI: 9.7-11.7). The 1-year survival rate was $42.1 \%$ and 2 -year survival rate was $12.2 \%$.

In the train cohort, patient clinical characteristics in the baseline and chemotherapy response-based models are summarized in Table 1 and Table S1(Supplement). For continuous predictors, median levels with interquartile ranges (IQRs) are provided in Table S2 (Supplement). The patient clinical characteristics of validation cohort are listed in TableS3 and TablsS4 (Supplement). Median levels of the continuous variables were close in the two models. Data distribution was similar between the baseline model and chemotherapy response-based model. In the chemotherapy responsebased model, 134 (57.5\%) patients were treated with nab-paclitaxel plus S1 chemotherapy regimen, 31 (13.3\%) patients were treated with gemcitabine monotherapy, $16(6.9 \%)$ patients were treated with nabpaclitaxel plus gemcitabine, 41 (17.6\%) patients were treated with gemcitabine-based combination chemotherapy, $4(1.7 \%)$ patients were treated with S1 monotherapy, and $7(3.0 \%)$ patients were treated with nab-paclitaxel monotherapy; 55 (23.6\%) patients showed progressive disease at the first evaluation after the second cycle of chemotherapy. Survival curves of different first-line chemotherapy regimens were shown in TableS5 and

Table 2 Results of univariate survival analysis

\begin{tabular}{|c|c|c|c|c|c|c|}
\hline \multirow[t]{2}{*}{ Characteristic } & \multicolumn{3}{|c|}{$\begin{array}{l}\text { Baseline model } \\
(N=323)\end{array}$} & \multicolumn{3}{|c|}{ Chemotherapy response-based model $(N=233)$} \\
\hline & HR & $95 \% \mathrm{Cl}$ & $p$ value & HR & $95 \% \mathrm{Cl}$ & $p$ value \\
\hline Gender (female vs male) & 1.45 & $1.10-1.92$ & 0.008 & 1.49 & $1.09-2.05$ & 0.011 \\
\hline Age $(\leq 56$ vs $>56)$ & 1.01 & $1.00-1.03$ & 0.012 & 1.22 & $0.90-1.66$ & 0.199 \\
\hline KPS $(70 \sim 80$ vs $90 \sim 100)$ & 0.57 & $0.43-0.77$ & $<0.001$ & 0.45 & $0.33-0.63$ & $<0.001$ \\
\hline Smoke (No vs Yes) & 1.32 & $1.00-1.74$ & 0.047 & 1.41 & $1.03-1.94$ & 0.034 \\
\hline Ascites (No vs Yes) & 1.46 & $1.08-1.96$ & 0.012 & 1.54 & $1.11-2.16$ & 0.011 \\
\hline Baseline WBC $(\leq 6.39$ vs $>6.39)$ & 1.63 & $1.25-2.13$ & $<0.001$ & 1.41 & $1.04-1.91$ & 0.027 \\
\hline Baseline $\mathbf{N}(\leq 0.665$ vs $>0.665)$ & 1.60 & $1.23-2.09$ & 0.001 & 1.68 & $1.24-2.29$ & 0.001 \\
\hline Baseline LDH ( $\leq 159$ vs > 159) & 1.38 & $1.06-1.80$ & 0.018 & 1.14 & $0.84-1.54$ & 0.402 \\
\hline Baseline TB $(\leq 11.4$ vs $>11.4)$ & 1.45 & $1.11-1.89$ & 0.006 & 1.43 & $1.06-1.94$ & 0.021 \\
\hline Baseline Alb ( $\leq 40.5$ vs > 40.5) & 0.76 & $0.58-1.00$ & 0.049 & 0.81 & $0.59-1.09$ & 0.166 \\
\hline Baseline CEA ( $\leq 7.32$ vs $>7.32$ ) & 1.47 & $1.13-1.92$ & 0.005 & 1.44 & $1.06-1.95$ & 0.019 \\
\hline Baseline CA 19-9 ( $\leq 1453$ vs > 1453) & 1.81 & $1.38-2.37$ & $<0.001$ & 1.72 & $1.26-2.34$ & 0.001 \\
\hline Change in CEA level at week 6 & & NI & & & & \\
\hline Decrease & & & & & Ref & \\
\hline No change or increase & & & & 1.39 & $1.02-1.91$ & 0.039 \\
\hline Change in CA19-9 level at week 6 & & NI & & & & \\
\hline Decrease & & & & & Ref & \\
\hline No change or increase & & & & 1.69 & $1.23-2.31$ & 0.001 \\
\hline Albumin at week 6 ( $\leq 39.0 \mathrm{vs}>39.0)$ & & $\mathrm{NI}$ & & 0.67 & $0.49-0.91$ & 0.010 \\
\hline TB at week $6(\leq 11.3 \mathrm{vs}>11.3)$ & & NI & & 1.36 & $1.01-1.85$ & 0.047 \\
\hline CEA at week 6 ( $\leq 7.97$ vs $>7.97)$ & & NI & & 1.58 & $1.17-2.14$ & 0.003 \\
\hline CA 19-9 at week 6 ( $\leq 790.7$ vs >790.7) & & NI & & 1.99 & $1.46-2.73$ & $<0.001$ \\
\hline Initial response to chemotherapy & & $\mathrm{NI}$ & & & & \\
\hline Non-PD & & & & & Ref & \\
\hline PD & & & & 3.07 & $2.17-4.35$ & $<0.001$ \\
\hline
\end{tabular}


Fig1S A-F (Supplement). The median overall survival of patients treated with nab-paclitaxel plus S1 was greater than gemcitabine monotherapy (9.92 months vs 6.18 months, $p=0.004)$. The effect of gemcitabine-based combination was superior to gemcitabine monotherapy (11.04 months vs 6.18 months, $p=0.019)$.

\section{Development of the nomogram prognostic model}

All demographic and clinicopathological variables, as well as tumor markers, were selected as candidate factors for the development of the prediction model. In the baseline model, twelve variables were selected for multivariable analysis in univariate survival analysis (Table 2 and Table S6, Supplement). Correlation analysis between twelve variables shown FigS2 (Supplement). In multivariable survival analysis, we used stepwise AIC backward regression to identify the six factors for the final prediction model: gender, KPS, baseline TB, baseline $\mathrm{N}$, baseline WBC and baseline CA19-9 (Table 3).

Similarly, in the chemotherapy response-based model, 16 variables showing statistical significance in univariate analysis were included in the correlation analysis. We excluded two variables (CA 19-9 level at 6 week and CEA level at 6 week) to decrease the influence on survival by multicollinearity (FigS3, Supplement). We used stepwise AIC backward regression to identify the nine factors for the final nomogram model: KPS, gender, ascites, baseline N, baseline CA 19-9, baseline CEA, change in CA 19-9 level at week, change in CEA level at week and initial response to chemotherapy (Table 3). Our results indicate that these nomograms can be used to evaluate the survival probability in patients with advanced and metastatic PC (Figs. 1 and 2).

\section{Nomogram validation}

For the baseline and chemotherapy response-based models, C-statistic, confirmed using 1000 bootstrap validation, were 0.67 (95\% CI, 0.62-0.71) and 0.74 (95\% CI, $0.69-0.77)$, respectively. Calibration curves also indicated good agreement between prediction and observation in the baseline and chemotherapy response-based nomogram models (Fig. 3A, B). In validation group, calibration curves demonstrated the good performance of two nomogram models (Fig. 3C-H). The accuracy of baseline and chemotherapy response-based nomogram model performed better in 2-year survival prediction than 1-year survival prediction. Predictive accuracy of the models with respect to individual and combined factors was compared using the ROC curves (Fig. 4A-D). Both in the train and validation group, the AUC of ROC curves could demonstrate the reliable of baseline and chemotherapy responsebased models.

\section{Clinical use}

We used X-tile to determine the optimal cut-off value in stratifying patients based on total scores

Table 3 Results of multivariate survival analysis

\begin{tabular}{|c|c|c|c|c|c|c|}
\hline \multirow[t]{2}{*}{ Characteristic } & \multicolumn{3}{|c|}{$\begin{array}{l}\text { Baseline model } \\
(N=323)\end{array}$} & \multicolumn{3}{|c|}{$\begin{array}{l}\text { Chemotherapy response-based model } \\
(N=233)\end{array}$} \\
\hline & $\mathrm{HR}$ & $95 \% \mathrm{Cl}$ & $p$ value & HR & $95 \% \mathrm{Cl}$ & $p$ value \\
\hline Gender (female vs male) & 1.71 & $1.33-2.19$ & $<0.001$ & 1.40 & $1.01-1.94$ & 0.039 \\
\hline KPS (70 80 vs 90 100) & 0.68 & $0.49-0.94$ & 0.018 & 0.57 & $0.40-0.80$ & 0.001 \\
\hline Ascites & & $\mathrm{NI}$ & & 1.47 & $1.04-2.08$ & 0.031 \\
\hline Baseline WBC & 1.55 & $1.17-2.06$ & 0.002 & & $\mathrm{NI}$ & \\
\hline Baseline N & 1.35 & $1.03-1.78$ & 0.032 & 1.56 & $1.14-2.13$ & 0.005 \\
\hline Baseline TB & 1.44 & $1.09-1.91$ & 0.012 & & $\mathrm{NI}$ & \\
\hline Baseline CA 19-9 & 1.87 & $1.41-2.47$ & $<0.001$ & 1.70 & $1.21-2.38$ & 0.002 \\
\hline Baseline CEA & & $\mathrm{NI}$ & & 1.54 & $1.09-2.19$ & 0.017 \\
\hline Change in CA19-9 level at week 6 & & $\mathrm{NI}$ & & & & \\
\hline Decrease & & & & & Ref & \\
\hline No change or increase & & & & 1.51 & $1.06-2.14$ & 0.017 \\
\hline Change in CEA level at week 6 & & $\mathrm{NI}$ & & & & \\
\hline Decrease & & & & & Ref & \\
\hline No change or increase & & & & 1.48 & $1.02-2.14$ & 0.039 \\
\hline Initial response to chemotherapy & & $\mathrm{NI}$ & & & & \\
\hline PD & & & & & Ref & \\
\hline Non-PD & & & & 0.46 & $0.31-0.69$ & $<0.001$ \\
\hline
\end{tabular}




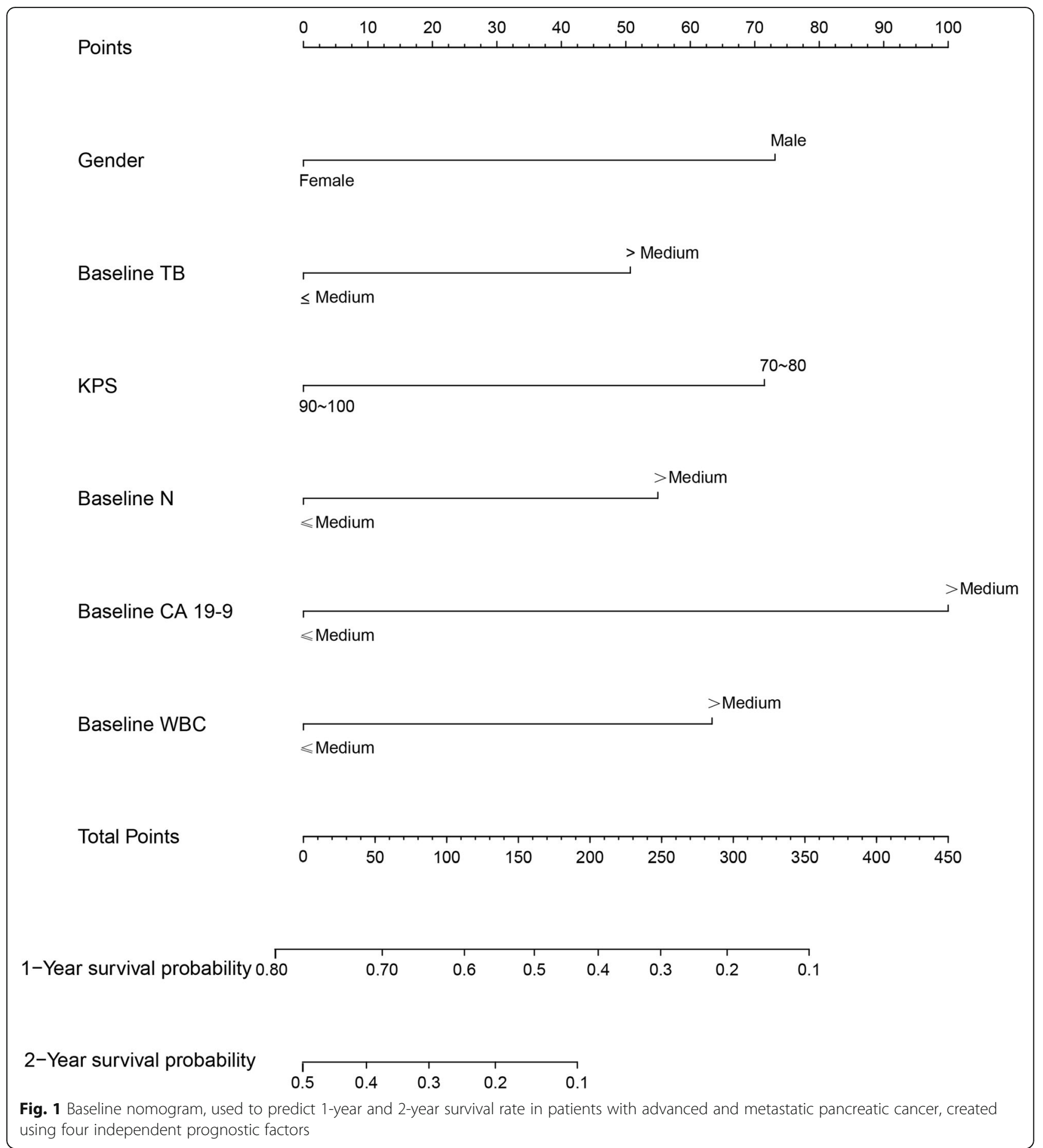

predicted by the nomograms; then, we plotted the Kaplan-Meier survival curves (Fig. 5A, B). The baseline model stratified at 340 scores and indicated increased survival time for patients with a total score higher than 340 (HR, 6.03; 95\% CI, 3.99-9.10; $p<$ 0.001). The chemotherapy response-based model stratified at 359 scores and indicated increased survival time for patients with scores higher than 359 (HR, 7.94; 95\% CI, 5.17-12.19; $p<0.001$ ).

We also used decision curve analysis (DCA) to evaluate the net benefit and clinical application value of our nomogram models. In the two nomogram models, the combined predictive models showed better clinical utility than the predictive value of any single variable (Fig. 6A-D). 


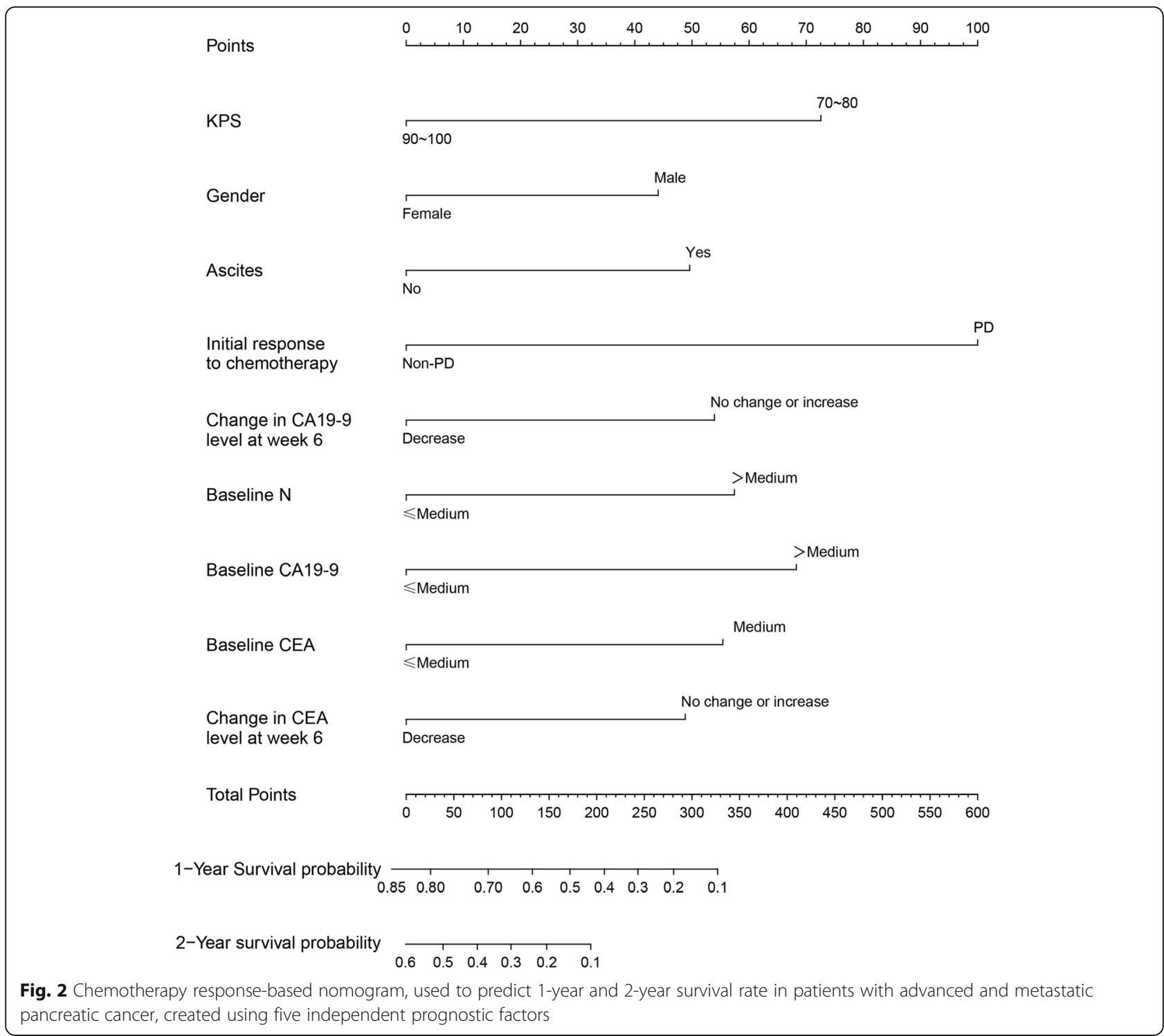

\section{Discussion}

In this study, we constructed two nomogram models that can be used to predict survival probability in patients with advanced and metastatic PC treated with first-line chemotherapy. Performance of the nomograms was rigorously assessed and internally validated. As shown by clinical trials, survival time of patients with advanced PC has gradually increased, but median overall survival remains less than 1 year with palliative chemotherapy [5]. Data on patient survival, estimated by prognostic models, can be used by physicians to prescribe suitable treatment and adjust therapies in a timely manner.

In our study, we used nomograms to predict the survival probability of patients with advanced PC. Our baseline nomogram, constructed using baseline clinical factors easily obtained before chemotherapy, showed good performance. This nomogram will aid physicians in making a preliminary survival assessment at the time of diagnosis and in prescribing appropriate dosage regimens. The chemotherapy response-based model was constructed after patients had undergone two cycles of chemotherapy. This model used factors such as initial response to chemotherapy and changes in the expression levels of tumor markers compared with those at baseline. This nomogram can be used to guide physicians in their decisions on whether to adjust treatment strategies.

In this study, we collected a wide array of variables previously reported to be associated with the prognosis of patients with PC $[20,21]$. Some of the risk factors associated with PC were also included in this study $[22,23]$. Previous studies have shown that changes in the levels of tumor markers are associated with survival of patients with advanced PC [24, 25]. For comprehensive assessment of patient survival, we collected 


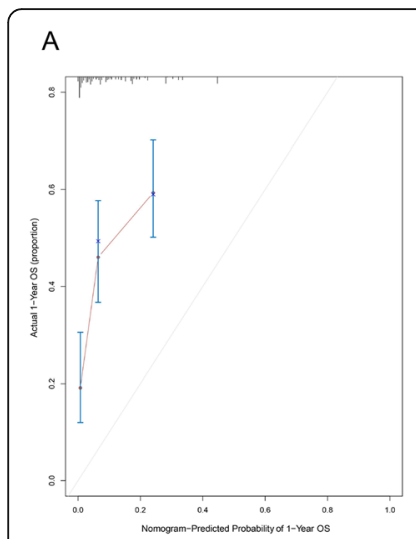

B

C
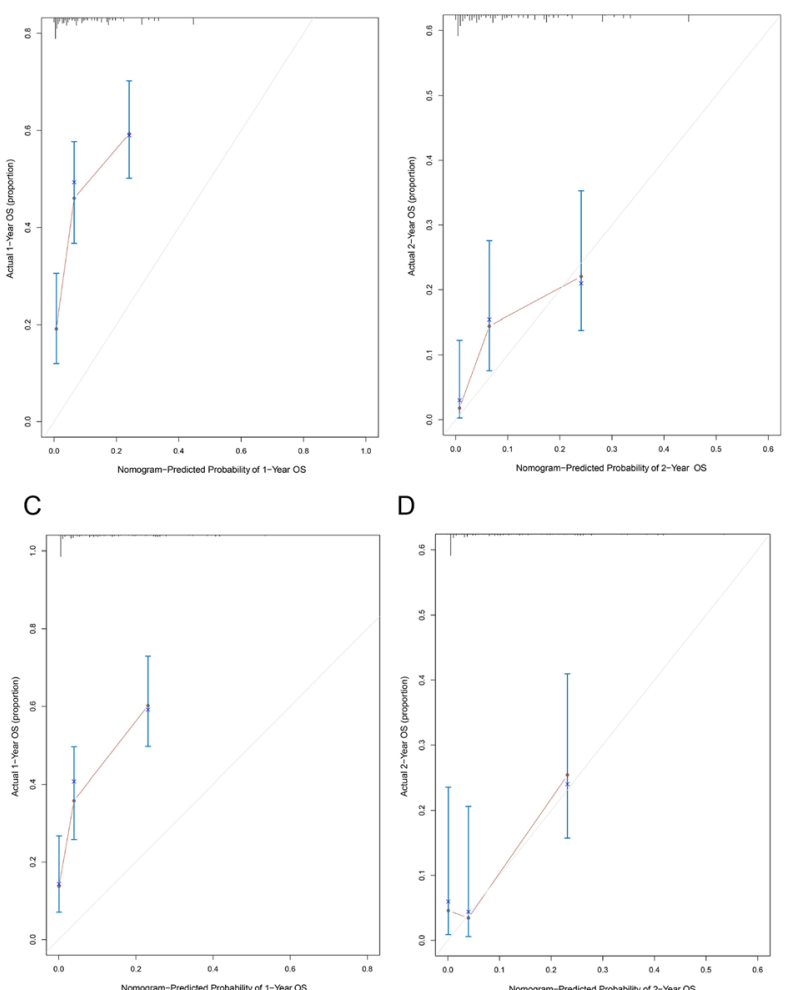

D

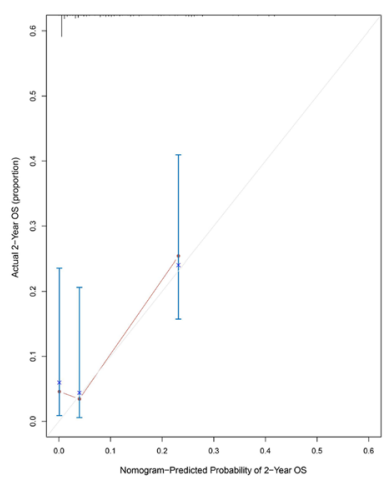

E

$\mathrm{F}$
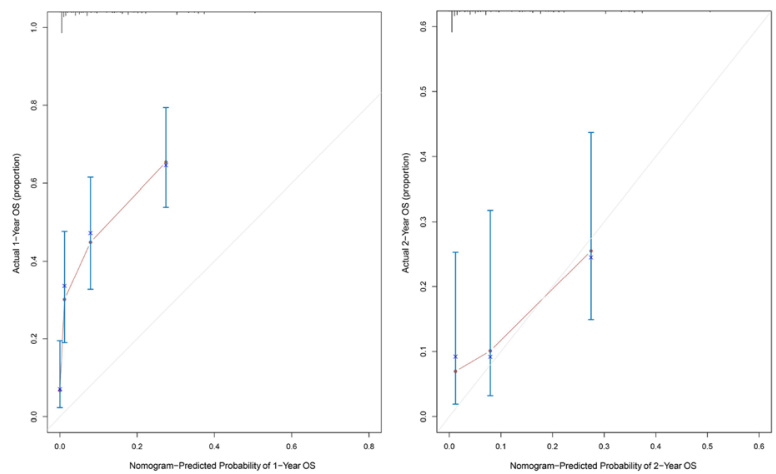

G

$\mathrm{H}$

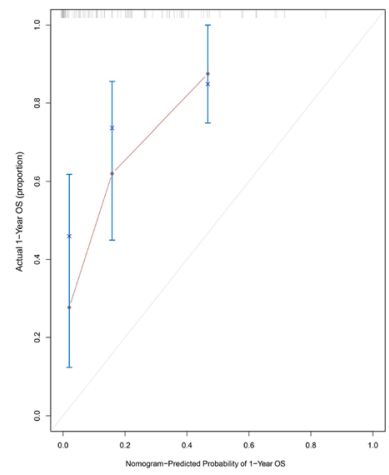

Fig. 3 Calibration plots of the two nomogram models comparing predicted probabilities against actual 1-year and 2-year survival rate. (A) Calibration plot of 1-year survival predictive in the baseline nomogram; (B) Calibration plot of 2-year survival predictive in the baseline nomogram; (C) Calibration plot of 1-year survival predictive in the chemotherapy response-based nomogram; (D) Calibration plot of 2-year survival predictive in the chemotherapy responsebased nomogram; (E) Calibration plot of 1-year survival predictive in external baseline validation group; (F) Calibration plot of 2-year survival predictive in external baseline validation group;(G)

Calibration plot of 1-year survival predictive in external chemotherapy validation group;(H) Calibration plot of 2-year survival predictive in external chemotherapy validation group

more factors than the numbers used in other studies. However, we did not incorporate all the variables into our model. After univariate analysis, we first used numerous association analyses to evaluate all the variables to avoid collinearity interference between variables. We then eliminated the variables having high correlation coefficients $(r>0.5, p<0.05)$. These variables have also been repeatedly validated in different studies, which helped us in locating and selecting pertinent information from large repositories of clinical data $[18,21]$.

Our baseline and chemotherapy response-based models demonstrated good discriminative ability, with C-statistic of 0.67 and 0.74 , respectively. The baseline model was similar to those constructed in previous studies $[21,26]$. In the two models constructed in our present study, baseline CA 19-9 level was an important factor for predicting the prognosis of patients with advanced PC. Previous studies have also shown that baseline CA 19-9 level is important in the construction of predictive models [20, 21, 27]. Because survival can be affected by the treatment process, we incorporated additional factors into our chemotherapy response-based model to improve the prognostic ability of the model; these factors included initial response to chemotherapy and expression levels of important laboratory markers. We found that in initial response to chemotherapy and changes in the levels of laboratory markers at 6 weeks were associated with survival probability, as shown by univariate analyses. We also performed Kaplan-Meier survival analyses of different first-line chemotherapy regimens. The results showed that the median overall survival of gemcitabine-based combination chemotherapy and nab-paclitaxel plus S1 was better than gemcitabine monotherapy with statistic significant (TableS5 and Fig1S A-F Supplement). Our team retrospectively analyzed multicenter first-line chemotherapy regimens of advanced pancreatic cancer, the results showed that nab-paclitaxel plus S1 was not inferior to nab-paclitaxel plus gemcitabine or FOLFIRINOX (5-fluorouracil, leucovorin, 

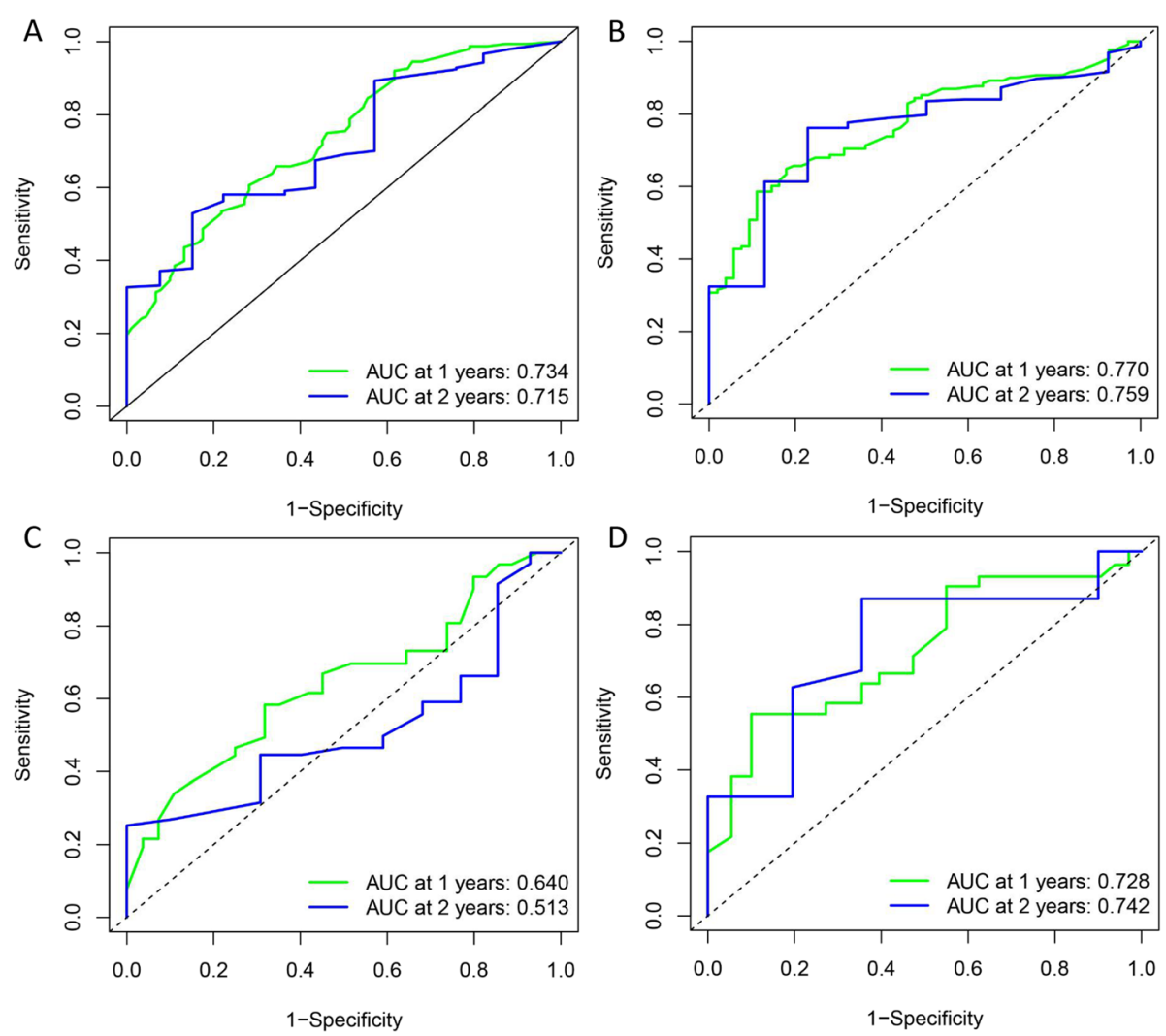

Fig. 4 Time-dependent receiver operating characteristic (ROC) curve was used to analyze the value of the nomograms in predicting 1-year and 2-year survival. (A) ROC curve of 1-year and 2-year survival predictive of baseline model in the train group; (B) ROC curve of 1-year and 2-year survival predictive of chemotherapy response-based model in the train group; (C)ROC curve of 1-year and 2-year survival predictive of baseline model in the validation group; (D) ROC curve of 1-year and 2-year survival predictive in the chemotherapy response-based model in the validation group
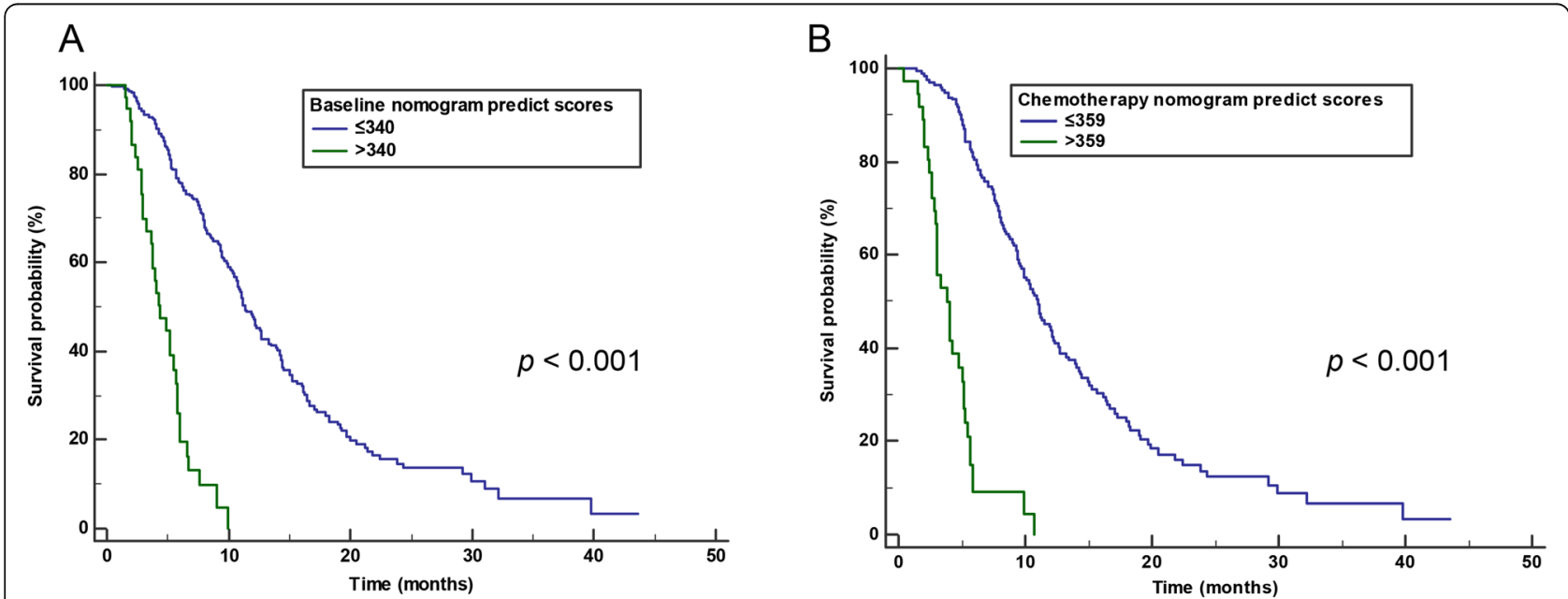

Fig. 5 Kaplan-Meier curves for patients stratified by the nomogram predictive scores. (A) Kaplan-Meier curves for patients evaluated using the baseline nomogram; (B) Kaplan-Meier curves for patients evaluated using the chemotherapy response-based nomogram 

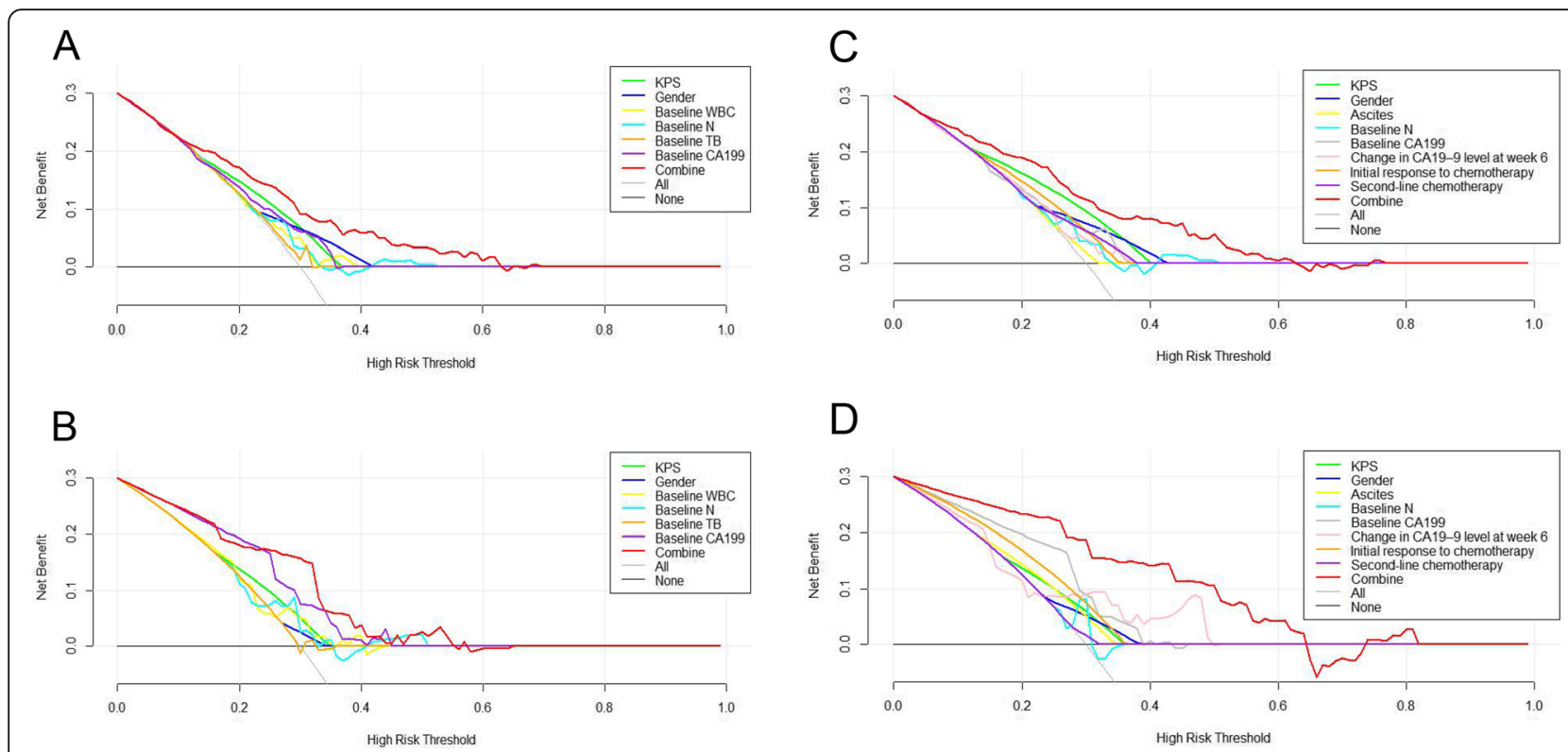

Fig. 6 Decision curve analysis (DCA) of the nomograms in prediction of 1-year and 2-year survival. The dark solid line represents the assumption that no patient survived beyond 1 or 2 year. The gray solid line represents the assumption that all patients survived in 1 or 2 year. The red solid line represents the combined nomogram. (A) DCA of 1-year survival predictive in the baseline nomogram; (B) DCA of 2-year survival predictive in the baseline nomogram; (C) DCA of 1-year survival predictive chemotherapy response-based nomogram; (D) DCA of 2-year survival predictive chemotherapy response-based nomogram

irinotecan, and oxaliplatin) [28]. However, the final model did not incorporate chemotherapy regimens or laboratory markers levels at 6 weeks, unless these changes were shown statistically significant by multivariate analysis. Our multivariate analysis indicated that the initial response to chemotherapy also was powerful in predicting patient survival. The initial response to chemotherapy can also be used to predict the response of other tumors to the chemotherapy being used [16, 29].

Our study had several limitations. Because it was a retrospective study, it may have been subject to biases. Additionally, our data were collected at a single center; therefore, in our future studies, we will obtain and combine data from multi-center databases to increase the credibility of our results. Finally, the patients evaluated in our study may have been subject to different subsequent treatment regimens. This factor, however, was not addressed in detail in our present study.

\section{Conclusions}

In this study, we used different clinical factors to construct nomograms that can be utilized to evaluate the survival probability of patients with advanced PC undergoing first-line chemotherapy. The baseline and chemotherapy response-based models developed in our present study showed good fit. Information obtained using these nomogram models can be used to assist clinicians in the selection and adjustment of treatment strategies. In our future studies, we will optimize these predictive models using multi-center data.

\section{Abbreviations}

KPS: Karnofsky performance status; CT: computed tomography; WBC: white blood cell; PLT: platelet; N: neutrophil; Alb: albumin; LDH: lactate dehydrogenase; TB: total bilirubin; CEA: carcinoembryonic antigen; CA 199: serum carbohydrate antigen 19-9; PD: progressive disease;

IQRs: interquartile ranges; VIF: Variance inflation factor; OS: overall survival; AIC: Akaike information criterion; C-index: concordance index; ROC: receiver operating characteristic; DCA: decision curve analysis; AUC: area under the curve

\section{Supplementary Information}

The online version contains supplementary material available at https://doi. org/10.1186/s12885-021-08943-w.

\footnotetext{
Additional file 1: Table S1. Patient characteristics of train groups (supplement data). Table S2. Patient Characteristics of train groups. Table S3. Patient characteristics of the validation groups. Table S4. Patient characteristics of validation groups. Table S5. Survival analysis of first-line chemotherapy regimens in train group. Table S6. Results of univariate survival analysis in train cohort (supplement data). Fig. S1. Kaplan-Meier survival curves of different chemotherapy regimens. A. Gem vs AS. B. Gem vs Gem-based. C. Gem vs AG. D. AS vs Gem-based. E. AS vs AG. F. Survival analysis of all chemotherapy regimens. Abbreviation: Gem: gemcitabine monotherapy; Gem-based: gemcitabine-based combination chemotherapy; AS: nab-paclitaxel plus S1; AG: nab-paclitaxel plus gemcitabine. Fig. S2. Correlation analysis between twelve survival-related variables in baseline group. Fig. S3. Correlation analysis between eighteen survival-related variables in chemotherapy group.
}

Acknowledgements

Not applicable. 


\section{Authors' contributions}

G.C Deng, Y LV and H Yan contributed equally as co-first authors. G.C Deng and Y LV participated in the main work. G.C Deng designed this study and drafted and revised this manuscript. G.C Deng and H Yan participated in acquisition of data. G.C Deng and Y LV participated in interpretation of data and analysis of data. H Yan, D.C Sun, T.T Qu and Y.T Pan participated in proofreading data. Q.L Han and G.H Dai are responsible for manuscript quality control and providing amendments. All authors read and approved the final manuscript.

\section{Funding}

Research was supported by the projects from National Natural Science Foundation of China (31671298). The funding body has no role in the design of the study, collection, analysis, interpretation of data, and writing of the manuscript.

\section{Availability of data and materials}

The data used to support the findings of this study are available from the corresponding author upon request.

\section{Declarations}

\section{Ethics approval and consent to participate}

Our study was approved by the ethics committee of PLA General Hospital. The ethical approval number is S2014-031-01. Clinical trial registration number is NCT02124317 (the first registration date: 28/4/2014). The first posted date is $28 / 4 / 2014$. All treatments were performed in accordance with institutional guidelines and regulations. Clinical data retrieved electronically from the medical records of PLA General Hospital Registry. Our study obtained informed consent from the study participants, in accordance with the Declaration of Helsinki.

\section{Consent for publication}

Not applicable.

\section{Competing interests}

The authors declare that they have no competing interests.

\section{Author details}

${ }^{1}$ School of Medicine, Nankai University, Tianjin, China. ${ }^{2}$ Senior Department of Oncology, The Fifth Medical Center of PLA General Hospital, Beijing, China.

\section{Received: 11 February 2021 Accepted: 28 October 2021}

Published online: 15 November 2021

\section{References}

1. Bray F, Ferlay J, Soerjomataram I, Siegel RL, et al. Global cancer statistics 2018: GLOBOCAN estimates of incidence and mortality worldwide for 36 cancers in 185 countries. CA Cancer J Clin. 2018;68(6):394-424.

2. WQ Chen, RS Zheng, Peter DB, et al. Cancer Statistics in China, 2015. CA Cancer J Clin. 2016.

3. Siegel RL, Miller KD, Jemal A. Cancer statistics, 2015. CA Cancer J Clin. 2015; 65(1):5-29. https://doi.org/10.3322/caac.21254

4. NCCN Guidelines Version 1.2019 Pancreatic Adenocarcinoma. Available from: https://www.nccn.org/professionals/physician_gls/pdf/pancreatic.

5. Gupta R, Amanam I, Chung V. Current and future therapies for advanced pancreatic cancer. J Surg Oncol. 2017;116(1):25-34. https://doi.org/10.1002/ jso.24623.

6. V.M. O, Chen Y, Babic A, Clish CB, Kraft P, Bao Y, et al. Cigarette smoking and pancreaticcancer survival. J Clin Oncol. 2017:35.

7. Yuan C, Bao Y, Wu C, Kraft P, Ogino S, Ng K, et al. Prediagnostic body mass index and pancreatic cancer survival. J Clin Oncol. 2013;31(33):4229-34. https://doi.org/10.1200/JCO.2013.51.7532.

8. Yuan C, Rubinson DA, Qian ZR, Wu C, Kraft P, Bao Y, et al. Survival among patients with pancreatic cancer and long-standing or recent-onset diabetes mellitus. J Clin Oncol. 2015;33(1):29-35. https://doi.org/10.1200/JCO.2014.57. 5688.

9. $\quad$ lic M, Ilic I. Epidemiology of pancreatic cancer. World J Gastroenterol. 2016; 22(44):9694-705. https://doi.org/10.3748/wjg.v22.i44.9694.
10. Jian-Guo Zhao YH, Liao Q, Niu Z-Y, Zhao Y-P. Prognostic significance of SUVmax and serum carbohydrate antigen 19-9 in pancreatic cancer.pdf. World J Gastroenterol. 2014;20:5875-80.

11. La Greca G, Sofia M, Lombardo R, Latteri S, Ricotta A, Puleo S, et al. Adjusting CA19-9 values to predict malignancy in obstructive jaundice: influence of bilirubin and C-reactive protein. World J Gastroenterol. 2012; 18(31):4150-5. https://doi.org/10.3748/wjg.v18.i31.4150.

12. F.T.S.K.R.C.F.S.B.S.R.D.D. Duranyildiz, Serum levels of LDH, CEA, and CA19-9 have prognostic roles on survival in patients with metastatic pancreatic cancer receiving gemcitabine-based chemotherapy.pdf. Cancer Chemother Pharmacol. 2014;73:1163-71.

13. Zhang J-X, Song W, Chen Z-H, Wei J-H, Liao Y-J, Lei J, et al. Prognostic and predictive value of a microRNA signature in stage II colon cancer: a microRNA expression analysis. The Lancet Oncology. 2013;14(13):1295-306. https://doi.org/10.1016/S1470-2045(13)70491-1.

14. Wang Y, Li J, Xia Y, Gong R, Wang K, Yan Z, et al. Prognostic nomogram for intrahepatic cholangiocarcinoma after partial hepatectomy. J Clin Oncol. 2013;31(9):1188-95. https://doi.org/10.1200/JCO.2012.41.5984.

15. Kim Y, Margonis GA, Prescott JD, Tran TB, Postlewait LM, Maithel SK, et al. Nomograms to Predict Recurrence-Free and Overall Survival After Curative Resection of Adrenocortical Carcinoma. JAMA Surg. 2016;151: 365-73.

16. Kim SY, Yoon MJ, Park YI, Kim MJ, Nam BH, Park SR. Nomograms predicting survival of patients with unresectable or metastatic gastric cancer who receive combination cytotoxic chemotherapy as first-line treatment. Gastric Cancer. 2018;21(3):453-63. https://doi.org/10.1007/s10120-017-0756-z.

17. Choi SH, Park SW, Seong J. A nomogram for predicting survival of patients with locally advanced pancreatic cancer treated with chemoradiotherapy. Radiother Oncol. 2018;129(2):340-6. https://doi.org/1 0.1016/j.radonc.2018.08.006.

18. Hang J, Wu L, Zhu L, Sun Z, Wang G, Pan J, et al. Prediction of overall survival for metastatic pancreatic cancer: development and validation of a prognostic nomogram with data from open clinical trial and realworld study. Cancer Med. 2018;7(7):2974-84. https://doi.org/10.1002/ca m4.1573.

19. Fornaro L, Leone F, Vienot A, Casadei-Gardini A, Vivaldi C, Lièvre A, et al Validated Nomogram Predicting 6-Month Survival in Pancreatic Cancer Patients Receiving First-Line 5-Fluorouracil, Oxaliplatin, and Irinotecan. Clinical Colorectal Cancer. 2019;18:e394-401.

20. Hamada T, Nakai $Y$, Yasunaga $H$, Isayama H, Matsui $H$, Takahara N, et al. Prognostic nomogram for nonresectable pancreatic cancer treated with gemcitabine-based chemotherapy. Br J Cancer. 2014;110(8):1943-9. https:// doi.org/10.1038/bjc.2014.131.

21. Zhang $L X$, Chen $L, X u$ AM. A simple model established by blood markers predicting overall survival after radical resection of pancreatic ductal adenocarcinoma. Front Oncol. 2020;10:583. https://doi.org/10.3389/fonc.202 0.00583.

22. Anderson MA, Zolotarevsky E, Cooper KL, Sherman S, Shats O, Whitcomb DC, et al. Alcohol and tobacco lower the age of presentation in sporadic pancreatic cancer in a dose-dependent manner: a multicenter study. Am J Gastroenterol. 2012;107(11):1730-9. https://doi.org/10.1038/ajg.2012.288.

23. Li D, Morris JS, Liu J, Hassan MM, Day RS, Bondy ML, et al. Body mass index and risk, age of onset, and survival in patients with pancreatic cancer. JAMA 2009;301(24):2553-62. https://doi.org/10.1001/jama.2009.886.

24. Chiorean EG, Von Hoff DD, Reni M, Arena FP, Infante JR, Bathini VG, et al. CA19-9 decrease at 8 weeks as a predictor of overall survival in a randomized phase III trial (MPACT) of weekly nab-paclitaxel plus gemcitabine versus gemcitabine alone in patients with metastatic pancreatic cancer. Ann Oncol. 2016;27(4):654-60. https://doi.org/10.1093/a nnonc/mdw006.

25. R.P. Michael H, Laubender, Petra S, Stefan H, Christiane J, Bruns, Ralf W, Ulrich M, Volker H, Stefan B, Prognostic relevance of CA 19-9, CEA, CRP, and $\mathrm{LDH}$ kinetics in patients treated with palliative second-line therapy for advanced pancreatic cancer. Tumor Biol.(2010) 31: 351-357.

26. Chen LT, Macarulla T, Blanc JF, Mirakhur B, Jong FA, Belanger B, et al. Nomogram for predicting survival in patients treated with liposomal irinotecan plus fluorouracil and Leucovorin in metastatic pancreatic Cancer. Cancers. 2019;11(8). https://doi.org/10.3390/cancers11081068.

27. B.G. Viviane H, Philipp G, Daniel D, György B, Thomas R, Emilio B, et al. CA19-9 tumour-marker response to chemotherapy in patients with 
advanced pancreatic cancer enrolled in a randomised controlled trial. Lancet Oncol. 2008;9:132-8.

28. Cui H, Guan J, Deng G, Yuan J, Lou C, Zhang W, et al. A Chinese retrospective multicenter study of first-line chemotherapy for advanced pancreatic Cancer. Med Sci Monit. 2020;26:e927654. https://doi.org/10.12 659/MSM.927654.

29. Piessevaux H, Buyse M, Schlichting M, Van Cutsem E, Bokemeyer C, Heeger $S$, et al. Use of early tumor shrinkage to predict long-term outcome in metastatic colorectal cancer treated with cetuximab. J Clin Oncol. 2013;31(30):3764-75. https://doi.org/10.1200/JCO.2012.42. 8532.

\section{Publisher's Note}

Springer Nature remains neutral with regard to jurisdictional claims in published maps and institutional affiliations.

Ready to submit your research? Choose BMC and benefit from:

- fast, convenient online submission

- thorough peer review by experienced researchers in your field

- rapid publication on acceptance

- support for research data, including large and complex data types

- gold Open Access which fosters wider collaboration and increased citations

- maximum visibility for your research: over $100 \mathrm{M}$ website views per year

At $\mathrm{BMC}$, research is always in progress.

Learn more biomedcentral.com/submissions 